\title{
Water vapor transmission properties of acrylic organic coatings
}

\author{
Réka Lilla Kovács, Lajos Daróczi, Péter Barkóczy, Eszter Baradács, Eszter Bakonyi, Szilvia Kovács, \\ Zoltán Erdélyi
}

Received: 16 April 2020/Revised: 27 August 2020/ Accepted: 31 August 2020

(C) The Author(s) 2020

\begin{abstract}
In this work, we evaluate the water vapor transmission rate (WVTR), the permeability $(P)$, solubility $(S)$, and diffusion $(D)$ coefficients of Paraloid B44, Paraloid B72, and Incralac coatings in the temperature range of $5-35^{\circ} \mathrm{C}$. The Arrhenius function-diffusion activation energy and preexponential factor-has also been determined from the data: $D_{B 44}=35.2 \mathrm{~cm}^{2} \mathrm{~s}^{-1} \exp \left(-25 \mathrm{~kJ} \mathrm{~mol}^{-1} / \mathrm{RT}\right) ; \quad D_{B 72}=$ $9.5 \mathrm{~cm}^{2} \mathrm{~s}^{-1} \exp \left(-23 \mathrm{~kJ} \mathrm{~mol}^{-1} / \mathrm{RT}\right) ; \quad D_{\text {Incralac }}=$ $622.8 \mathrm{~cm}^{2} \mathrm{~s}^{-1} \exp \left(-28 \mathrm{~kJ} \mathrm{~mol}^{-1} / \mathrm{RT}\right)$. These resins are important coating materials, for example, for conservators to protect metallic artifacts, such as statues, against corrosion. Despite Paraloid B44 and $\mathrm{B} 72$ resins being considered as reference materials in conservation practice, that is, new coating materials (either water vapor retarders or transmitters) are often compared to them, there are no comprehensive data for the quantities describing the vapor permeability $(P$, $S, D)$ of these materials. The measurements are based on the ISO cup-method using substrate/coating composite samples. The strength of this technique is that it can also be used when the coating is non-self-support-
\end{abstract}

R. L. Kovács ( $₫)$, L. Daróczi, E. Baradács,

Z. Erdélyi

Department of Solid State Physics, Faculty of Sciences and

Technology, University of Debrecen, P.O. Box 400,

Debrecen 4002, Hungary

e-mail: kovacs.reka@science.unideb.hu

P. Barkóczy

FUX Zrt, Miskolc, Hungary

E. Bakonyi

Hungarian National Museum, Budapest, Hungary

S. Kovács

Department of Agricultural Botany, Plant Physiology and Biotechnology, Faculty of Agricultural and Food Sciences and Environmental Management, University of Debrecen, Debrecen, Hungary ing; nevertheless, $P, S$, and $D$ can be deduced for the coating layer itself, and it seems to be a standardizable procedure for comparative performance testing of coating materials. Paraloid B72 layers exhibited higher WVTRs-from 39 to $315 \mathrm{~g} \mathrm{~m}^{-2}$ day $^{-1}$ as the temperature increased from 5 to $35^{\circ} \mathrm{C}$-compared to Paraloid B44 and Incralac coatings-from 17 to $190 \mathrm{~g} \mathrm{~m}^{-2}$ day $^{-1}$, respectively. The transmission rate parameters were also compared to the results of corrosion tests. Incralac was the most effective corrosion inhibitor, and the performance of the B44 was better than the B72, which is in good agreement with the transmission rate tests.

Keywords Acrylic resins (Paraloid B44 and B72, Incralac), Water vapor transmission rate (WVTR) measurement, Water vapor permeability $(P)$ and solubility $(S)$, Diffusion coefficient of water vapor $(D)$, Activation energy of water vapor diffusion

\section{Introduction}

Several kinds of polymeric resins are used in the art conservation practice, mainly in conservation and bonding. The main question is in all cases the protection efficiency of the resins against the deterioration of the objects. One of the most important tasks is the corrosion protection of cultural heritage. ${ }^{1-3}$ Protective coatings made of these resins are applied to prevent corrosion. ${ }^{4,5}$ It is, however, also necessary to take into consideration the visual appearance and visualization of the objects in addition to corrosion protection. This principle poses a restriction on the applied coatings, mainly the type and the thickness of resins. The size and the complexity of the shape of the object determine the coating method. Acrylic polymers, such as Paraloid B72, Paraloid B44, and Incralac, are largely used, for example, in art conservation because of their 
reversibility, long-term stability, easy applicability (they have negligible harmful volatile emission), good adhesion to the substrate, and hydrophobicity. Conservators apply them as coatings, consolidants, or adhesives. Moreover, Paraloid B44 and B72 are used as references when introducing new materials in the conservation practice. ${ }^{6-18}$ The available water vapor permeability data of these materials, such as water vapor transmission rate (WVTR) or moisture vapor transmission rate (MVTR), permeability coefficient $(P)$, solubility or absorptivity coefficient $(S)$, and diffusion coefficient $(D)$, scatter significantly and are measured under different conditions. Generally, they are not determined for the coating itself, but WVTR is only measured for the substrate/coating composites with various resins and these data are used for the coating qualification. In order to provide useful and reliable data, we have determined all these quantities in the temperature range of $5-35^{\circ} \mathrm{C}$.

The aim of most of the coatings in art conservation is to protect surfaces of artifacts, such as statues, paintings, buildings, and organic materials like wood and paper. One of the most important physical properties of the coatings is the water vapor transmission rate (WVTR). ${ }^{5,11,19-24}$ In some cases, low WVTR, and in other cases, high WVTR is desired. For example, in the case of wall paintings, the water inside them exchanges with the outer environment in the form of vapor through the porous structure. The hindering of this exchange by low WVTR coatings leads to the gathering of soluble salts, causing deterioration of wall paintings. ${ }^{24,25}$ In the case of metal artifacts, however, complete isolation from the environment (corrosion resistance) is preferred; that is, in the latter case, the application of low VWTR coatings is required.

Obviously, the water vapor transmission of the coatings cannot be studied on the original object. First, the valuable objects cannot be involved in experiments; second, it requires too much time to see the result, i.e., the signs of corrosion. Accordingly, there are two possibilities to study the effectiveness of the coatings, that is, their WVTR. Either we choose a substrate material (mainly metals) to hold the coating and we measure its corrosion, or we try to measure the WVTR of the coating directly. The first method is a test mimicking the real situation, however, it is very time consuming even in the case of accelerated aging. ${ }^{26}$ The second method is much faster, as we will demonstrate in this work, and accordingly provides the possibility to test a huge number of coatings in a reasonable time. For this reason, a good practice can be to test the resins by using the latter method to select the best candidates, and then do the final selection with the first method (i.e., accelerated corrosion testing of coatings on metal surfaces), if necessary.

Many published works produce useful stand-alone data, which usually compare coatings using highly specific goals and customized testing regimes to deal with the multiple numbers of variables within the test procedures. There remains a need for developing test procedures that support conservation aims and enable comparative performance testing of coating materials. $^{26}$ The present work introduces a test procedure that supports conservation goals and facilitates comparative performance testing of coating materials. The evidence-based information obtained from the experiments could be helpful for professionals working with these materials both in museums and industrial fields.

The measurements are based on the ISO cupmethod using substrate/coating composite samples. The strength of this technique is that it can also be used when the coating is non-self-supporting; nevertheless, $P, S$, and $D$ can be deduced for the coating layer itself. From the temperature dependence of these quantities, by applying the well-known Arrhenius equation, we give the activation energies and the preexponential factors for water diffusion in Paraloid B44, Paraloid B72, and Incralac resins.

To perform a real-life test, we subjected metallic samples to a standardized salt solution immersion corrosion test and investigated them using a scanning electron microscope equipped with an energy-dispersive spectrometer (SEM/EDX).

Accordingly, the paper is organized as follows: In the Materials and methods section, we describe the materials and the experimental methods we used. In the Calculations section, we show how the permeability and solubility coefficients of the coating layer-in our case, resin-can be calculated if we measure the WVTR and the solubility of a substrate and the substrate/coating layer composite. Using these, the diffusion coefficient can then be calculated. In the Results and discussion section, we summarize the experimental findings and give the values of the coefficients at different temperatures. Then, we show the results of the corrosion tests. The paper closes with the Conclusions section.

\section{Materials and methods}

\section{Materials}

Three different, frequently used resins, Paraloid B44 (Kremer Pigmente), Paraloid B72 (Kremer Pigmente), and Incralac (Kremer Pigmente) have been compared. The resins are originally solids; therefore, they must be dissolved in different solvents (e.g., acetone, xylol, petrol) to make them suitable for application. In our case, the resins were dissolved in acetone (Honeywell, assay minimum $99.5 \%$ ) with different concentrations: $15 \mathrm{~g}$ Paraloid B44 and B72 dissolved in $100 \mathrm{ml}$ acetone, and $40 \mathrm{~g}$ Incralac dissolved in $100 \mathrm{ml}$ acetone, that is, 15 and 40 weight/volume percent (w/v \%) solutions, respectively. Laminated paper $\left(40 \mathrm{~g} \mathrm{~m}^{-2}\right.$ paper laminated with $20 \mathrm{~g} \mathrm{~m}^{-2}$ low-density polyethylene) was used as a substrate. ${ }^{27}$ 


\section{Methods}

\section{Coating techniques}

In practice, the applied coating technique depends on the size and shape of the metallic object, and on the properties of the resin. Immersion and brushing are routinely used by the conservators, but these methods are not suited for a standardized certification procedure. As a consequence, it was necessary to develop a method for layer preparation to build the coating with appropriate quality and, very importantly, reproducibility; that is, the coating layer has to be uniform. Accordingly, we used a purpose-built spin-coating machine to coat the substrate membranes having a surface area of $25 \mathrm{~cm}^{2}$. The necessary speed of rotation was determined experimentally prior to the cup preparation. This speed depends on the viscosity of the solution and the wetting between the substrate and the solution. In our experiments, $2500 \mathrm{rpm}$ was used for the resin solutions. This trial is necessary in all cases when a new resin is tested, or a new solution is used.

The WVTR depends not only on the coating technique and the material of the coating but also on its thickness. Therefore, the surface coverage, homogeneity, and thickness of the resin layers on test samples were analyzed by optical microscopy (Zeiss Axio Scope.A1, Carl Zeiss $\mathrm{GmbH}$ ), by scanning electron microscopy (HITACHI S4300-CFE, Hitachi High-Technologies Europe $\mathrm{GmbH}$ ) and by monitoring the mass increment of the substrate with an analytical balance (KERN ADB 200-4, KERN \& SOHN $\mathrm{GmbH})$. The reproducibility of the thickness is also a key issue. As the substrates had a fixed surface, we always dropped the same volume of solution on the substrate surface by an automatic pipette to deposit the same amount of material, hence the same thickness. In the case of the Paraloid B44 and B72, $0.6 \mathrm{ml}$ solution was dropped, whereas three times $0.6 \mathrm{ml}$ was dropped for the Incralac, which resulted in a layer thickness of $3 \mu \mathrm{m}( \pm 3 \%)$. The thickness was determined from the surface area, the density (Paraloid B44: $1174 \mathrm{~kg} \mathrm{~m}^{-3}$, Paraloid B72: $1150 \mathrm{~kg} \mathrm{~m}^{-3}$, Incralac: $899 \mathrm{~kg} \mathrm{~m}^{-3}$ ), and the mass of the resins obtained as the difference in the coated and uncoated substrates. From every test run, one coated sample was also checked with microscopic techniques before the tests to make sure that the full surface of the substrate was covered with the resin layer, and to double-check the calculated thickness. These results confirmed the precise surface coverage and thicknesses.

To examine if the results obtained for the coatings prepared by the spin coating can be applied in general, we also used brushing as it is the most frequently used technique in art conservation. ${ }^{23}$ In this case, we dropped $0.6 \mathrm{ml}$ on the substrate surface with an automatic pipette, and the whole surface of the substrate was covered by using a soft brush. The thicknesses of the coating layers were $10 \mu \mathrm{m}( \pm 20 \%)$. The reproducibility of the layer depends on the practice and experience of the person who prepares the layer. On the other hand, it gives the possibility to evaluate the protective properties of coatings in which conditions are closer to the ones made in practice. This fact causes higher uncertainty values for coatings prepared by brushing. Nevertheless, since the coatings are brushed on plane surfaces during the tests, it is expected that sufficiently homogeneous layers can be built for testing purposes.

\section{Water vapor transmission rate measurement}

The water vapor transmission rate is measured on the basis of the standardized cup method EN ISO 7783-1. ${ }^{28}$ The procedure consists of the following steps. Some amount of hygroscopic material, $15 \mathrm{~g} \mathrm{CaCl}_{2}$ (Honeywell Chemicals, assay minimum $97.0 \%$ ), is placed in a cup covered by the membrane of which WVTR is to be determined. On the top of the membrane, a ring template, with an inner diameter of $5.64 \mathrm{~cm}$, defines the exact test surface. Mechanical clamping seals the sample to the dish. The whole system is kept in controlled atmosphere: constant pressure, ambient humidity, and temperature. The amount of water vapor flowing into the cup through the membrane and absorbed by the hygroscopic material is deduced as a function of time by measuring the increase in mass of the cup-membrane-hygroscopic material set-up by an analytical balance in regular time intervals. To increase the accuracy of the measurements, five parallel tests were performed in each experiment.

Knowing the exposed surface of the membrane $A$, $25 \mathrm{~cm}^{2}$, the water vapor transmission rate can be calculated as follows:

$\mathrm{WVTR}=\frac{\Delta W}{A \Delta t}\left[\mathrm{~g} \mathrm{~m}^{-2} \mathrm{day}^{-1}\right]$

where $\Delta W$ is the mass of the absorbed water during time $\Delta t^{28-35}$

The mass of the absorbed water by the $\mathrm{CaCl}_{2}$ was measured with high accuracy by the KERN ADB 200-4 analytical balance.

The constant temperature and relative humidity were provided by a climate chamber (Xi'an LIB Environmental Simulation Industry, model TH-50A).

\section{Immersion corrosion test}

To determine the corrosion resistance of the acrylic resins, a laboratory immersion corrosion test, based on the ASTM G31-72 standard, was performed. ${ }^{36,37}$ Homogenous $30 \% \mathrm{Zn}$ and $70 \% \mathrm{Cu}$ brass samples (dimensions: $14 \mathrm{~mm}$ width, $26 \mathrm{~mm}$ height, $1 \mathrm{~mm}$ thickness) were cleaned with deionized water and brushed with $0.2 \mathrm{~mL}$ of $15 \mathrm{w} / \mathrm{v} \%$ Paraloid B44, B72 and $40 \mathrm{w} /$ v\% Incralac solutions. For thickness measurement, a 
part of the coating was removed with acetone, and the height profile was measured with an Ambios XP-1 profilometer. The coating thickness was $20( \pm 3)$ microns for each sample. Three pieces of coated brass sheets for each resin type and three uncoated samples were then placed in a glass flask with a supporting device which ensured that the brass sheets were kept fully submerged in the $5 \mathrm{wt} \% \mathrm{NaCl}$ (Lach-ner, assay minimum $99.5 \%$ ) solution in distilled water during the 7 days of exposure time. The tests were conducted at $35^{\circ} \mathrm{C}$.

After the corrosion test, the samples were cleaned and then analyzed by a HITACHI S4300-CFE scanning electron microscope equipped with a Bruker energy-dispersive X-ray detector (SEM/EDX).

\section{Measurement of the solubility}

The gravimetric method was chosen to measure the water uptake to determine the solubility or absorptivity coefficient in this work. ${ }^{24,38-40}$ The method is based on the measurement of the weight gain of the sample in contact with water vapor at constant temperature and pressure and calculated as:

$\hat{S}=\frac{M_{\infty}}{v \cdot p}$,

where $M_{\infty}$ is the total mass of vapor absorbed by the polymer sample at equilibrium, $v$ is the volume of the sample under test, and $p$ is the permeant driving force.

\section{Calculation}

\section{Permeability}

\section{Homogeneous membranes}

Knowing the water vapor transmission rate, we can also determine the permeability coefficient used often to describe the diffusion rate through a membrane. The basic equation for diffusion is Fick's first equation:

$j=-D \frac{\partial \varrho}{\partial x}$

where $j$ is the flux of the diffusion species, $D$ is the diffusion coefficient, $\varrho$ is the volume density (concentration). ${ }^{41}$ Combining this equation and the equation of the conservation of matter, we obtain Fick's second equation, which has the following form if we consider that the diffusion coefficient is independent of the space coordinate:

$\frac{\partial \varrho}{\partial t}=D \frac{\partial^{2} \varrho}{\partial x^{2}}$.
In steady state, $\frac{\partial \varrho}{\partial t}=0$ everywhere and any time, accordingly

$0=D \frac{\partial^{2} \varrho}{\partial x^{2}}$,

that is,

const $=D \frac{\partial \varrho}{\partial x}$

Comparing this to equation (2), we may see that the flux $j$ is constant and thus integrating $j$ from 0 to $l$ along the $x$ direction, we get the following expression: $j l=-D\left(\varrho_{2}-\varrho_{1}\right)$, which we can write in a more convenient form as follows:

$j=-D \frac{\varrho_{2}-\varrho_{1}}{l}$,

where $\varrho_{1}$ and $\varrho_{2}$ are the concentrations of the diffusion species on the surface of the membrane. In the case of diffusion of vapor through a membrane, the surface concentrations $\varrho_{1}$ and $\varrho_{2}$ may not be known but only the vapor pressure $p_{1}$ and $p_{2}$ on the two sides of the membrane. If the diffusion coefficient is constant, and if the sorption isotherm is linear, that is, there is a linear relationship between the external vapor pressure and the corresponding equilibrium concentration within the membrane:

$\varrho=S p$,

we may write equation (6) as:

$j=-P \frac{p_{2}-p_{1}}{l}$,

where $S$ is the solubility, $P$ is the permeability coefficient [17]:

$P=D S$.

As the flux $j$ measures the number of diffusing species that will flow through a unit area of the membrane during a unit time interval, the WVTR, which measures the mass of transported species that will flow through a unit area of the membrane during a unit time interval, can be calculated by multiplying equation (8) by the mass $m$ of the diffusing species:

$W V T R=-m P \frac{p_{2}-p_{1}}{l}$.

In our experiments, the vapor pressures on the two sides of the membrane, $p_{1}$ and $p_{2}$, are not known, only the values of the relative humidity $R H$. By definition, the relative humidity is the ratio of the partial pressure of water vapor to the equilibrium vapor pressure, $p_{\mathrm{e}}$; 
the WVTR at a given temperature can be expressed in terms of $R H$ (see equation (10)):

$W V T R=-m P p_{e} \frac{\frac{p_{2}}{p_{e}}-\frac{p_{1}}{p_{e}}}{l}=-m P p_{e} \frac{R H_{2}-R H_{1}}{l}$.

As the relative humidity in the cap, $R H_{2}$, is zero, the permeability can be calculated as follows:

$P=\frac{W V T R l}{m p_{\mathrm{e}} R H_{1}}$

\section{Composite membranes}

Using equation (12), we can determine the permeability of either the substrate or the substrate/resin composite membrane. We are, nevertheless, interested in the permeability of the resin. As we will see below, this can be deduced if we measure the permeability of both the substrate and the composite membrane.

In general, a composite membrane consists of $n$ layers (submembranes) with thickness $l_{i}$. The permeability, the diffusion coefficient, and the solubility in each layer are $P_{i}, D_{i}$, and $S_{i}$; moreover, the difference in concentration on the two sides of the submembranes is $\Delta \varrho_{i}$, respectively (see Fig. 1 ).

We may calculate the flux of water vapor in each layer by using equation (6):

$j_{i}=-D_{i} \frac{\Delta \varrho_{i}}{l_{i}}$.

Using the relationships given in equations (7) and (9), we get:

$j_{i}=-P_{i} \frac{\Delta p_{i}}{l_{i}}$

for $i=1, \ldots, n$, where $\Delta p_{i}$ is the difference in pressure on the two sides of the submembranes. Since the rate of transfer is the same across each submembrane, i.e., $j_{i}=j$, the fall in pressure in one layer is:
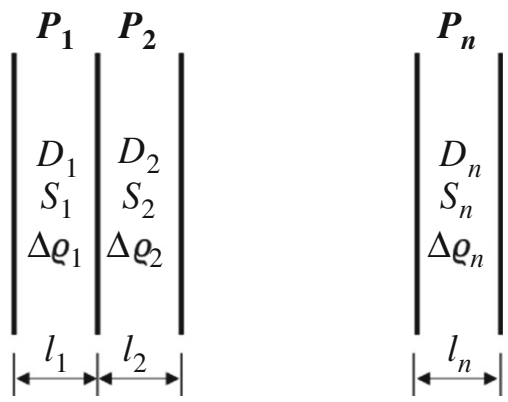

Fig. 1: Scheme of a composite membrane consisting of $n$ layers
$\Delta p_{i}=-j \frac{l_{i}}{P_{i}}$

The total fall in pressure in the whole membrane is obviously:

$\Delta p=\sum_{i=1}^{n} \Delta p_{i}=-j \sum_{i=1}^{n} \frac{l_{i}}{P_{i}}$

For the whole membrane, we may write:

$\Delta p=-j \frac{l}{P}$

where $l=\sum_{i=1}^{n} l_{i}$ is the total thickness of the membrane. Comparing equations (16) and (17), we get:

$\frac{l}{P}=\sum_{i=1}^{n} \frac{l_{i}}{P_{i}}$

Therefore, in the case of a substrate/resin composite membrane, we have:

$\frac{l_{\mathrm{r}}}{P_{\mathrm{r}}}=\frac{l}{P}-\frac{l_{\mathrm{s}}}{P_{\mathrm{s}}}$,

where the subscripts ' $r$ ' and ' $s$ ' denote the resin and the substrate, respectively, from which $P_{\mathrm{r}}$ can easily be calculated.

\section{Solubility}

\section{Homogeneous membranes}

The $\hat{S}$ water solubility coefficient determined by gravimetric method described in the "Measurement of the solubility" section is a measure of the mass of permeant molecules absorbed by a unit volume of the sample per unit of partial pressure; accordingly, its unit is $\mathrm{kg} \mathrm{m}^{-3} \mathrm{~Pa}^{-1}$. (See equation (1)). The definition of the solubility $S$ in equation (7) is, however, a bit different, as $S$ is a measure of the number of permeant molecules sorbed by a unit volume of the sample per unit of partial pressure, and so its unit is $1 \mathrm{~m}^{-3} \mathrm{~Pa}^{-1}$. Therefore, obviously, $\hat{S}$ divided by the mass of the absorbed molecule $m$ equals $S$ :

$S=\frac{\hat{S}}{m}$ 


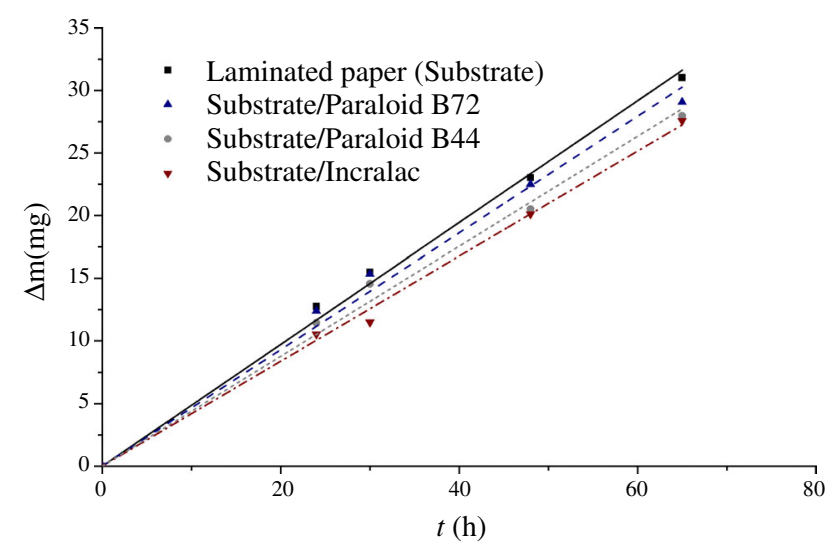

Fig. 2: The mass increment as a function of time measured at $20^{\circ} \mathrm{C}$; the slopes of the fitted lines divided by the area of the samples give the WVTR values. Each data point represents the average of the values measured in five identically prepared samples. The length of the error bar is comparable to the size of the symbols

\section{Composite membranes}

In the case of a composite membrane, shown in Fig. 2, only the overall solubility coefficient of the whole composite can be directly measured. The solubility coefficient of the substrate (homogeneous membrane) can, however, also be directly measured. In the case of a binary composite membrane, e.g., a substrate/resin layer in our case, these two data provide us with the possibility to calculate the solubility coefficient in the resin.

According to equation (7), the solubility coefficient can be expressed as the ratio of the volume density of the water molecules in the sample and the pressure (permeant driving force): $S=\rho / p$. The density of water molecules in the substrate/resin composite can be calculated as:

$\varrho=\frac{M_{\infty}}{v \cdot m}=\frac{\varrho_{\mathrm{s}} m A l_{\mathrm{s}}+\varrho_{\mathrm{r}} m A l_{\mathrm{r}}}{A\left(l_{\mathrm{s}}+l_{\mathrm{r}}\right) m}$,

where $\varrho_{\mathrm{s}}$ and $\varrho_{\mathrm{r}}$ are the volume density (its unit is $1 \mathrm{~m}^{-3}$ ) of the water molecules in the substrate and resin, respectively, and $A$ is the cross-sectional area of the substrate, hence also the resin layer. Dividing equation (21) by $p$ and considering that $S=\rho / p$, we get:

$S=\frac{S_{\mathrm{s}} l_{\mathrm{s}}+S_{\mathrm{r}} l_{\mathrm{r}}}{l_{\mathrm{s}}+l_{\mathrm{r}}}$.

As the parameters $S, S_{\mathrm{S}}, l_{\mathrm{s}}$, and $l_{\mathrm{r}}$ can be directly measured, $S_{\mathrm{r}}$ can be calculated.

\section{Diffusion coefficient}

To determine the diffusion coefficients in a coating layer, that is, in the resin in our case, the procedure is the following: measurement of the WVTR and the solubility coefficients for both the substrate and the substrate/resin layer composite; calculation of their permeability coefficients by equation (12); calculation of the permeability coefficient of the resin by using equation (19); calculation of the solubility in the resin by equation (22); and calculation of the diffusion coefficient in the resin by using the relationship given by equation (9).

As is well known, the temperature dependence of the diffusion coefficient follows the Arrhenius law ${ }^{41}$ :

$D=D_{0} \exp \left(-\frac{Q}{R T}\right)$

where $D_{0}$ is the preexponential factor, $Q$ is the activation energy, $R$ is the molar gas constant, and $T$ is the absolute temperature. Accordingly, by measuring the diffusion coefficient at different temperatures, $D_{0}$ and $Q$ can be calculated.

\section{Results and discussion}

\section{Water vapor transmission rate, solubility and diffusion coefficient}

As shown in "Calculation" section, to determine the permeability, the solubility, and the diffusion coefficient of the resins, we need to measure the total water uptake $\left(M_{\infty}\right)$ and to determine the WVTR for the laminated paper (substrate) and the laminated paper covered by the given resin (substrate/resin composite). As an example, Fig. 2 shows the mass increment of the samples in time measured at $20^{\circ} \mathrm{C}$, of which the slope divided by the area of the sample yields the values of WVTR of the substrate/resin composite. The values of $M_{\infty}$ and WVTR for all samples and temperatures are given in the "Appendix Table A1."

Figure 3 shows the WVTR, permeability $(P)$, solubility $(S)$, and diffusion $(D)$ coefficients of the resins calculated as described in the "Calculation" section (see also "Appendix Table A2"). Note that the thickness of the substrate was $60 \mu \mathrm{m} ; m$ was calculated as the ratio of the molar mass of water, and Avogadro's constant $\left(m=M_{H_{2} O} / N_{A}\right)$ and the equilibrium water vapor pressure values were taken from reference (42).

Using the diffusion coefficient data, we may construct an Arrhenius function. The plot of the data points is shown in Fig. 4 for the three different resins, and Table 1 contains the values of the preexponential factor and the activation energy.

We repeated these measurements and evaluation of the data when brushing was used as the coating technique. As the layers prepared by brushing were 

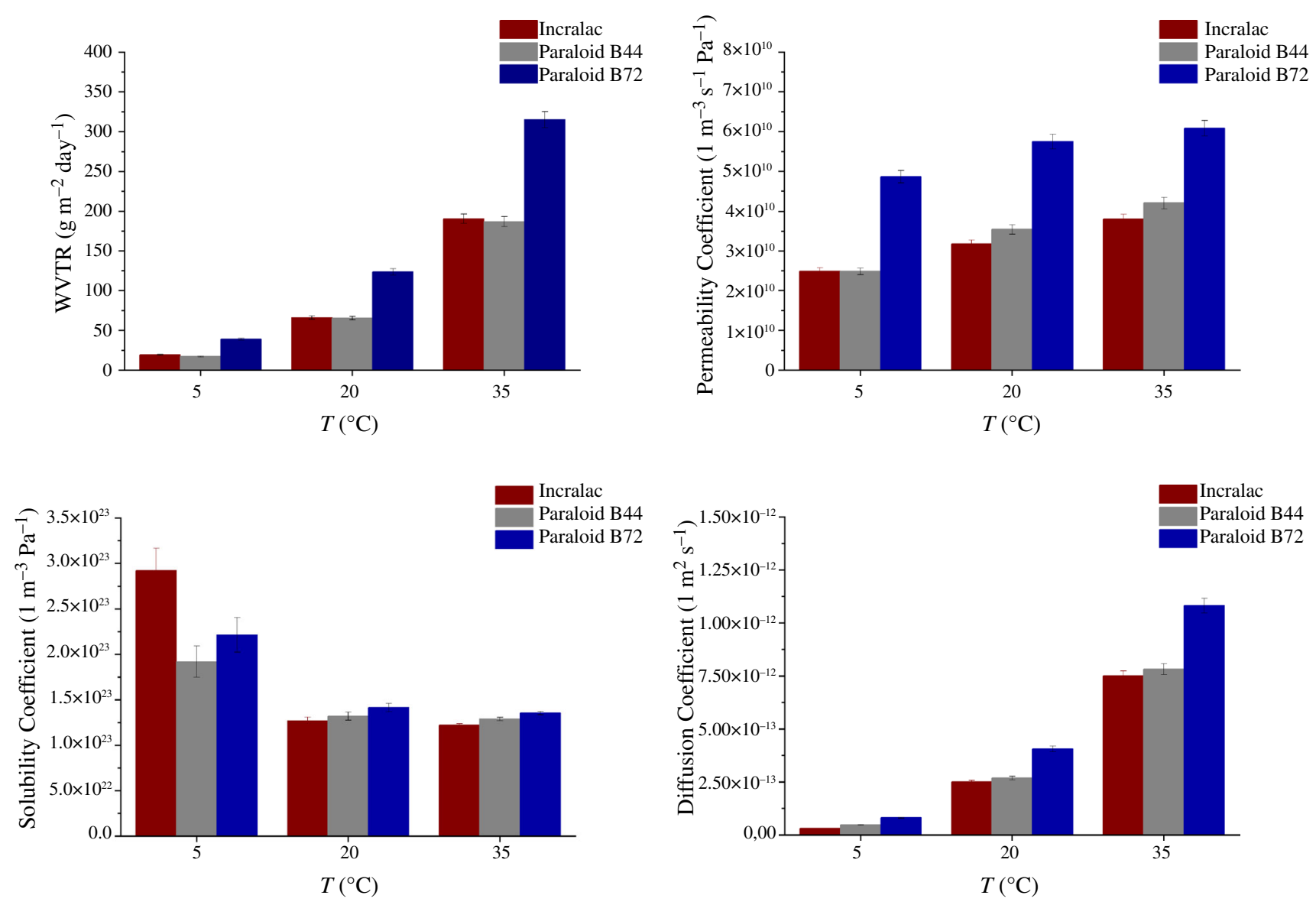

Fig. 3: The calculated WVTR, permeability $(P)$, solubility/absorptivity $(S)$, and diffusion (D) coefficients of the Paraloid B44, $\mathrm{B72}$, and Incralac layers at different temperatures. The error bars of the values were calculated from the standard error of the layer thickness measurements and from the standard error of the fitting parameters

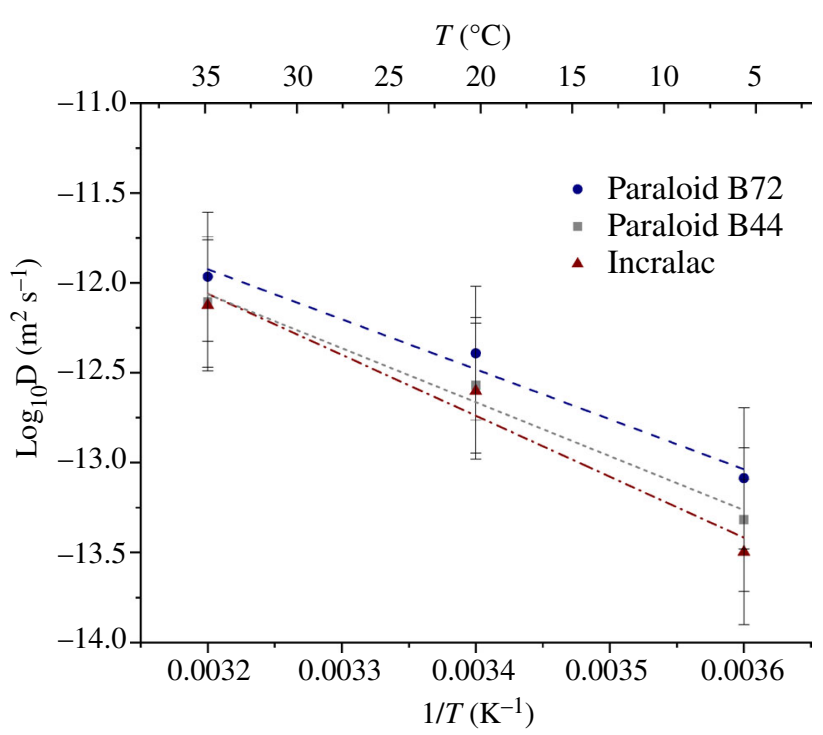

Fig. 4: Arrhenius plot of the data points for the Paraloid B44 (square), B72 (spot), and the Incralac (triangle) resins. A straight line is fitted to the data points from which slope and intercept the activation energy and the preexponential factors can be determined. (Table 1) about three times thicker $(10 \mu \mathrm{m} \pm 20 \%)$ than by spin coating $(3 \mu \mathrm{m} \pm 3 \%)$, consequently their WVTR values were lower, around the half of the data obtained by spin coating. The deduced $P$ and $D$ values were, however, close to those obtained from the spin-coating experiments (within a factor of two). This confirms that the highly reproducible spin-coating technique is suitable for the tests, which is not only more accurate, but requires much less practice to use than other coating techniques.

Using these data, and furthermore knowing the average relative humidity of the place where the object to be protected against corrosion (e.g., artifact, sculptures) is located, the surface area of the object and the water vapor exposure time, we may estimate the difference in the total amount of water vapor reaching the object with or without protective coating.

For example, the surface area of a realistic, fulllength figure of a person is about $2 \mathrm{~m}^{2}$. If we consider an art conservation period of 20-25 years for these objects, this means that an object protected by the Paraloid B72 is affected by $1809-2261 \mathrm{~L}$ of water, whereas in the case of Paraloid B44 or Incralac resins, only about 958-1197 or 967-1209 L, respectively. This 
Table 1: Diffusion activation energy and preexponential factors for the different resins determined from the Arrhenius plot in Fig. 4

\begin{tabular}{lcc} 
Resin & Water vapor diffusion activation energy $\left(\mathrm{kJ} \mathrm{mol}^{-1}\right)$ & Water vapor diffusion preexponential factor $\left(\mathrm{cm}^{2} \mathrm{~s}^{-1}\right)$ \\
\hline Paraloid B44 & $25 \pm 0.7$ & $35.2 \pm 1.2$ \\
Paraloid B72 & $23 \pm 0.7$ & $9.5 \pm 0.3$ \\
Incralac & $28 \pm 0.8$ & $622.8 \pm 19.3$
\end{tabular}

The uncertainty of the values was calculated from the standard error of fitting parameters and of the layer thickness measurements

implies that, for instance, by choosing the Paraloid B72 instead of the Paraloid B44, the excess of water reaching the object is about $851-1064 \mathrm{~L}$, which must result in much enhanced corrosion. Furthermore, when Paraloid B72 is used as a consolidant, the WVTR of the coating is also a key factor as salts and pollutants could easily dissolve in water and attack the structure of the valuable object. ${ }^{18}$ This means that extensive study of the applied resins in art conservation practice is highly worthwhile.

The deduced quantitative data must be valuable not only for conservation practice, especially since these data are hardly available in the literature since usually only the WVTR is measured for comparison purposes; that is, a given substrate is coated with different resins, and then the WVTR of the substrate/coating composites is measured and compared to qualify the coatings. The quantities describing the vapor permeability (WVTR, $P, D$ ) are not deduced for the coatings itself.

For example, we only found two works of which data could be used to estimate these quantities for the Paraloid B72. Carretti et al. ${ }^{11}$ and Zhang et al. ${ }^{23}$ measured the WVTR of plaster and mortar samples coated with Paraloid B72. Their aim was to show that the new coating materials they studied had much higher WVTR than that of the Paraloid B72, which is favorable to protect wall paintings. So, they did not determine the $P$ or $D$, and did not even calculate the WVTR for the Paraloid B72 coating itself. From their data, we could, however, estimate the permeability coefficient of Paraloid B72, which is $6.68 \times 10^{12} \mathrm{~m}^{-1}$ $\mathrm{s}^{-1} \mathrm{~Pa}^{-1} \cdot{ }^{11}$ This value is about two orders of magnitude greater than what we obtained $\left(5.75 \times 10^{10} \mathrm{~m}^{-1} \mathrm{~s}^{-1}\right.$ $\mathrm{Pa}^{-1}$ at $20^{\circ} \mathrm{C}$ ). However, we have to consider that plaster and mortar are porous materials. Accordingly, the substrate/coating composite also suffers from certain discontinuity, which obviously influences the WVTR. On the contrary, the laminated paper has a highly even surface, and so the coating resin forms a perfect continuous film, as can be seen in Fig. 5a.

To see how the morphology of the substrate, hence that of the coating, influences the transmission properties, we repeated our measurements with a substrate having a significantly rougher structure than that of the laminated paper. As Fig. 5b shows, the impregnated paper is a purposeful choice to mimic the porous-like structure of plaster and mortar-still having a paper as a substrate to be able to perform the measurements without any modification for better comparison. The transmission measurements, indeed, showed an increase in permeability value by an order of magnitude $\left(6.57 \times 10^{11} \mathrm{~m}^{-1} \mathrm{~s}^{-1} \mathrm{~Pa}^{-1}\right.$ at $\left.20^{\circ} \mathrm{C}\right)$.

From these literature data, however, we cannot estimate the diffusion coefficient and the activation energy, because, on one hand, the authors did not provide solubility data, and on the other hand, they performed measurements only at one temperature. We can, however, compare our results to that obtained in polymethyl methacrylate (PMMA), which is the main component of the Paraloid B44. This shows that the diffusion activation energy we deduced seems to be highly reasonable. The literature value varies in the range of $23.9-63.2 \mathrm{~kJ} \mathrm{~mol}^{-1}$. ${ }^{43}$

\section{Immersion corrosion test}

The water vapor transmission rate measurement data were compared to outcomes in a standardized salt solution immersion corrosion test. The homogenous $70 / 30$ brass sheets were coated with the three different resins by brushing. After exposing the coated and uncoated samples in the solution for 7 days, the zinc and oxygen contents were measured by SEM/EDX at different positions to study and reveal the difference in the corrosion effect.

As an example, Fig. 6 shows the typical SEM pictures of the covered brass sheets after the salt solution treatment. The SEM/EDX measurements showed that the zinc content on the surface of the B72-treated sample decreased the most significantly, and hardly changed in the case of the B44 and Incralac. Similarly, the oxygen content increased the most in the case of the B72 coating, much less in the case of the B44, and practically did not change for the Incralac. The extent of the affected areas and the average zinc content clearly show that the Paraloid B44 performs better in this test than the B72, which is in agreement with the transmission rate measurements. In the case of Incralac-coated samples, almost no changes can be detected. Although the transmission rate data of the B44 and the Incralac are very similar, the Incralac provides better protection against corrosion. According to the literature, this is related to its 

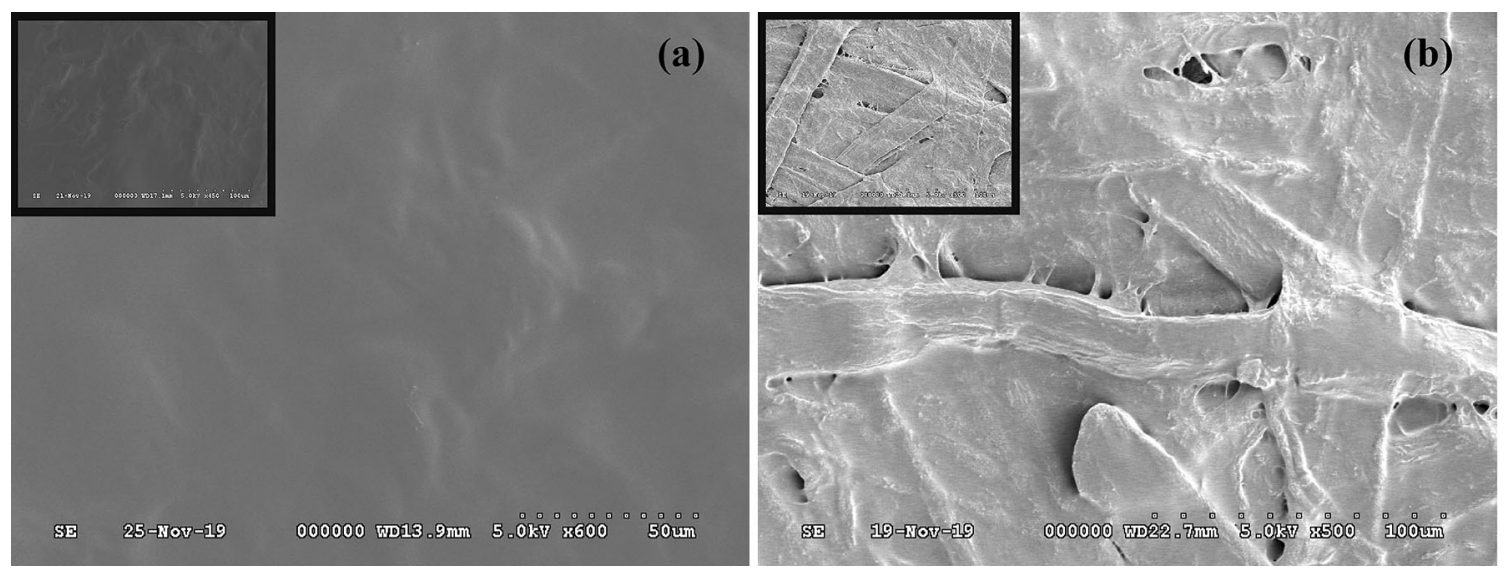

Fig. 5: SEM micrographs of the substrates coated with acrylic resins. Smooth and continuous layer can be formed on the surface on the laminated paper (a). Impregnated paper (b) is more porous as well as the resin film, which causes higher WVTR and permeability of this composite system. The insets show the surface of the uncoated substrates
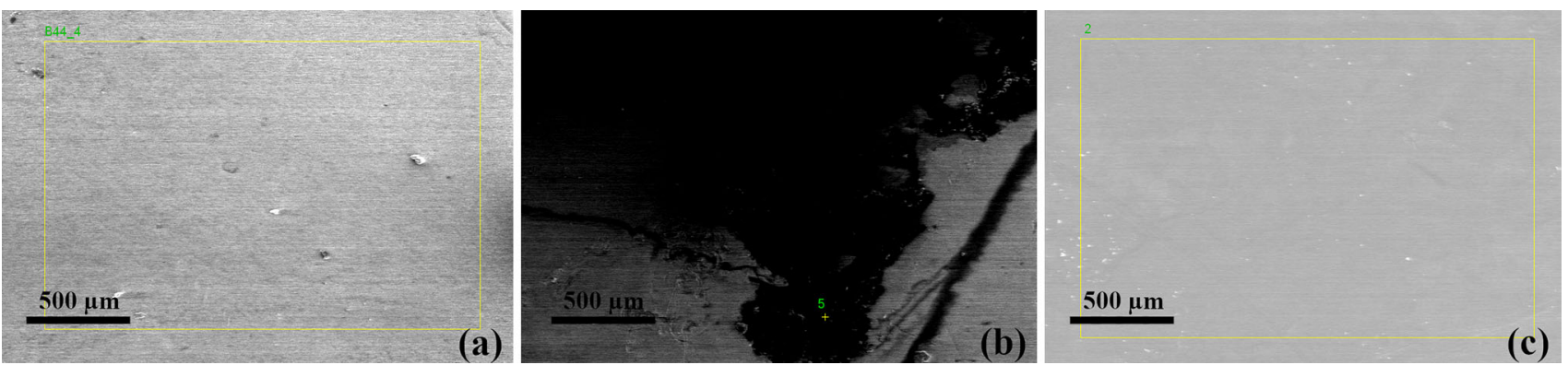

Fig. 6: SEM micrographs of the salt solution immersion tested brass samples. The Paraloid B44 (a) and Incralac (c)-coated samples show moderate homogenous corrosion. On the B72-treated (b) surface, however, extensive corrosion occurred. In the black areas, extremely low zinc and high oxygen concentrations can be measured

benzotriazole (BTA) content, which is an effective corrosion inhibitor for copper alloys. BTA derivatives adsorb onto the brass surface and form a protective complex film that sufficiently prevents corrosion; that is, the Incralac not only isolates the brass from the salt solution but also passivates the surface. ${ }^{4-48}$ The water vapor transmission and diffusion parameters are "intrinsic" quantities, and the presented method can be used to evaluate these data for coating materials. Although, in different operational environments, the coating performance could be altered compared to the transmission data because of the substrate/coating interactions.

\section{Conclusions}

Despite the fact that the Paraloid B44 and Paraloid B77 resins are reference materials, that is, new coating materials are often compared to them qualitatively, there are no comprehensive data for the quantities describing the vapor permeability $(P, S, D)$ of these materials. In this work, using our transmission rate measurement techniques based on the ISO cupmethod, we determined these values in the temperature range of $5-35^{\circ} \mathrm{C}$ and calculated the diffusion activation energy and the preexponential factors (Arrhenius function) for these resins and also the Incralac, which is also frequently used in conservation practice. The introduced method seems to be a standardizable comparative testing technique for coating materials (or techniques) in conservation practice since the layer quality, thickness, and homogeneity of the coating are independent of the operator's practice, hence highly reproducible. Employing this procedure, the intrinsic transmission properties of the resins can be determined, which makes it easier to evaluate which resin is the most beneficial (e.g., life span, cost, ease of use) for a given purpose, when either high or low vapor transmission rate is required. Our results could be useful guidelines for conservators to choose the appropriate coating layer for different materials, i.e., wood, metal, stone, etc. which are in different operating environments.

With an immersion corrosion test, we showed that the sample coated by the Paraloid B44 is much less 
affected by corrosion than the sample coated by the Paraloid B72 resin. This excellent agreement shows that the procedure we developed can be used to predict the protection efficiency of resin layers with a high degree of realism. The Incralac, which was developed for $\mathrm{Cu}$-based alloys as a corrosion inhibitor, also showed very good protective properties, even better than the Paraloid B44, although their transmission parameter values hardly differ. This has been explained by its BTA content, which passivates the brass surface.

We emphasize here again that this transmission rate experimental method we used to qualify the coatings is much less time consuming and more cost-effective than the conventional corrosion tests, like traditional corrosion tests + SEM/EDX. This makes it possible to perform a large number of tests. Moreover, the transmission rate experimental method is highly quantitative, which facilitates the decision of what coating method and material is the most beneficial and costeffective in a given situation-not exclusively in art conservation practice.

Although the method in this work was used to study resins as coating material, the routine can be applied in a variety of applications, for example, for substrate/coating composites, when the coating is nonself-supporting, but the quantitative data for the coating itself is required.

Acknowledgments The work is supported by the GINOP-2.3.2-15-2016-00041 project. The project is cofinanced by the European Union and the European Regional Development Fund. We must express our appreciation to Dr. Gábor Langer for sharing his

wisdom with us during this research and for moderating this paper and in that line improving the manuscript significantly. We thank Dr. Gábor Erdélyi for the constructive suggestions on the technical content and grammar. We would like to express our deep gratitude to Ágnes Bonivárt, from the School of Computer Science, University of Hertfordshire for her diligent proofreading.

Open Access This article is licensed under a Creative Commons Attribution 4.0 International License, which permits use, sharing, adaptation, distribution and reproduction in any medium or format, as long as you give appropriate credit to the original author(s) and the source, provide a link to the Creative Commons licence, and indicate if changes were made. The images or other third party material in this article are included in the article's Creative Commons licence, unless indicated otherwise in a credit line to the material. If material is not included in the article's Creative Commons licence and your intended use is not permitted by statutory regulation or exceeds the permitted use, you will need to obtain permission directly from the copyright holder. To view a copy of this licence, visit http://creativecommons.org/licenses/by/4.0/.

Funding Open access funding provided by University of Debrecen.

\section{Appendix}

Table A1: Measured water vapor transmission rate (WVTR) and total mass of absorbed vapor $M_{\infty}$ for the laminated paper (substrate) and for substrate/resin coating composites

\begin{tabular}{lcrr} 
& $\mathrm{T}\left({ }^{\circ} \mathrm{C}\right)$ & WVTR $\left(\mathrm{g} \mathrm{m}^{-2}\right.$ day $\left.^{-1}\right)$ & $M_{\infty}(\mathrm{mg})$ \\
\hline Laminated paper (substrate) & 5 & $2.10 \pm 0.13$ & $4.87 \pm 0.31$ \\
Substrate/Paraloid B44 & 5 & $1.74 \pm 0.05$ & $5.17 \pm 0.30$ \\
Substrate/Paraloid B72 & 5 & $1.93 \pm 0.06$ & $5.40 \pm 0.25$ \\
Substrate/Incralac & 5 & $1.78 \pm 0.06$ & $5.10 \pm 0.10$ \\
Laminated paper (substrate) & 20 & $4.52 \pm 0.14$ & $3.27 \pm 0.17$ \\
Substrate/Paraloid B44 & 20 & $4.06 \pm 0.13$ & $3.47 \pm 0.30$ \\
Substrate/Paraloid B72 & 20 & $4.26 \pm 0.14$ & $3.67 \pm 0.31$ \\
Substrate/Incralac & 20 & $4.06 \pm 0.13$ & $2.82 \pm 0.25$ \\
Laminated paper (substrate) & 35 & $12.98 \pm 0.65$ & $2.50 \pm 0.26$ \\
Substrate/Paraloid B44 & 35 & $11.63 \pm 0.38$ & $3.50 \pm 0.29$ \\
Substrate/Paraloid B72 & 35 & $12.15 \pm 0.39$ & $3.07 \pm 0.15$ \\
Substrate/Incralac & 35 & $11.66 \pm 0.36$ & $2.70 \pm 0.10$ \\
\hline
\end{tabular}

The uncertainty of the values was calculated from the standard error of the slope and the uncertainty of the weight measurement 
Table A2: Calculated WVTRs, permeability, solubility/absorptivity, and diffusion coefficients for the resins

\begin{tabular}{lrrrrr} 
& $\mathrm{T}\left({ }^{\circ} \mathrm{C}\right)$ & WVTR $\left(\mathrm{g} \mathrm{m}^{-2} \mathrm{day}^{-1}\right)$ & $P\left(\mathrm{~m}^{-1} \mathrm{~s}^{-1} \mathrm{~Pa}^{-1}\right) \times 10^{9}$ & $S\left(\mathrm{~m}^{-3} \mathrm{~Pa}^{-1}\right) \times 10^{23}$ & $D\left(\mathrm{~m}^{2} \mathrm{~s}^{-1}\right) \times 10^{-14}$ \\
\hline Paraloid B44 & 5 & $17.14 \pm 0.57$ & $24.89 \pm 0.83$ & $5.16 \pm 0.17$ & $4.82 \pm 0.16$ \\
Paraloid B44 & 20 & $65.48 \pm 2.18$ & $35.45 \pm 1.18$ & $1.32 \pm 0.04$ & $26.91 \pm 0.90$ \\
Paraloid B44 & 35 & $186.94 \pm 6.23$ & $42.04 \pm 1.40$ & $0.54 \pm 0.02$ & $78.24 \pm 2.61$ \\
Paraloid B72 & 5 & $39.03 \pm 1.25$ & $48.66 \pm 1.56$ & $5.94 \pm 0.19$ & $8.19 \pm 0.26$ \\
Paraloid B72 & 20 & $123.67 \pm 3.96$ & $57.49 \pm 1.84$ & $1.42 \pm 0.05$ & $40.61 \pm 1.30$ \\
Paraloid B72 & 35 & $315.30 \pm 10.10$ & $60.89 \pm 1.95$ & $0.56 \pm 0.02$ & $108.22 \pm 3.47$ \\
Incralac & 5 & $19.37 \pm 0.60$ & $24.98 \pm 0.78$ & $7.84 \pm 0.24$ & $3.19 \pm 0.10$ \\
Incralac & 20 & $66.15 \pm 2.05$ & $31.80 \pm 0.99$ & $1.27 \pm 0.04$ & $25.06 \pm 0.78$ \\
Incralac & 35 & $190.63 \pm 5.92$ & $38.08 \pm 1.18$ & $0.51 \pm 0.02$ & $75.07 \pm 2.33$ \\
\hline
\end{tabular}

\section{References}

1. Funke, W, "Problems and Progress in Organic Coatings Science and Technology." Prog. Org. Coat., 31 (1-2) 5-9 (1997)

2. Werner, A, "Synthetic Materials in Art Conservation." $J$. Chem. Educ., 58 (4) 321-324 (1981)

3. Horie, CV, Materials for Conservation-Organic Consolidants: Adhesives and Coatings. Architectural Press, Butterworth-Heinemann, Oxford (1987)

4. Boyatzis, S C, Veve, A, Kriezi, G, Karamargiou, G, Kontou, E, Argyropoulos, V, “A Scientific Assessment of the LongTerm Protection of Incralac Coatings on Ancient Bronze Collections in the National Archaeological Museum and the Epigraphic and Numismatic Museum in Athens, Greece." In: Daehner, J M (ed.) Artistry in Bronze The Greeks and Their Legacy XIXth International Congress on Ancient Bronzes, pp. 300-311. The J. Paul Getty Museum and the Getty Conservation Institute, Los Angeles (2017)

5. Funke, W., "Mechanisms of Protecting Metals by Organic Coatings Against Corrosion." In: Kendig, MW, Leidheiser $\mathrm{H}, \mathrm{Jr}$ (ed.) Corrosion Protection by Organic Coatings, Electrochemical Proceedings Volume 87-2, The Electrochemical Society Inc., Pennington, NJ (1987)

6. Švadlena, J, Stoulil, J, "Evaluation of Protective Properties of Acrylate Varnishes Used for Conservation of Historical Metal Artefacts." Koroze A Ochrana Material, 61 25-31 (2017)

7. Kapolos, J, Bakaoukas, N, Koliadima, A, Karaiskakis, G, "Evaluation of Acrylic Polymeric Resin and Small Siloxane Molecule for Protecting Cultural Heritage Monuments Against Sulfur Dioxide Corrosion." Prog. Org. Coat., 59 152-159 (2007)

8. Farmakalidis, H, Douvas, A, Karatasios, I, Sotiropoulou, S, Boyatzis, S, Argitis, P, Chryssoulakis, Y, Kilikoglou, V, "Accelerated Thermal Ageing of Acrylic Copolymers, Cyclohexanone-based and Urea-aldehyde Resins Used in Paintings Conservation." Mediterr. Archaeol. Archaeom., 16 213-228 (2016)

9. Bruhin, S, Hildbrand, E, Sangouard, E, Schramm, J, "Protection of Organic Remains in Alkaline Iron Desalination." In: ICOM-CC 18th Triennial Conference, Copenhagen (2017), ISBN: 9789290124269

10. Yang, L, Wang, L, Wang, P, "Investigation of Photo-stability of Acrylic Polymer Paraloid B72 Used for Conservation." Sciences of Conservation and Archaeology, 19 54-58 (2007)
11. Carretti, E, Dei, L, "Physicochemical Characterization of Acrylic Polymeric Resins Coating Porous Materials of Artistic Interest.” Prog. Org. Coat., 49 (3) 282-289 (2004)

12. Wolfe, J, Grayburn, R, "A Review of the Development and Testing of Incralac Lacquer.' J. Am. Inst. Conserv., 56 (3-4) 225-244 (2017)

13. Koob, S, "The Use of Paraloid B-72 as an Adhesive: Its Application for Archaeological Ceramics and Other Materials." Stud. Conserv., 31 7-14 (1986)

14. Toniolo, L, Paradisi, A, Goidanich, S, Pennati, G, "Mechanical Behaviour of Lime Based Mortars After Surface Consolidation." Const. Build. Mat., 25 1553-1559 (2011)

15. Ćurković, H, Kosec, T, Marušić, K, Legat, A, “An Electrochemical Impedance Study of the Corrosion Protection of Artificially Formed Patinas on Recent Bronze." Electrochim. Acta, 83 28-39 (2012)

16. Constâncio, C, Franco, L, Russo, A, Anjinho, C, Pires, J, Vaz, MF, Carvalho, A, "Studies on Polymeric Conservation Treatments of Ceramic Tiles with Paraloid B-72 and Two Alkoxysilanes." J. App. Poly. Sci., 116 (5) 2833-2839 (2010)

17. Vinçotte, A, Beauvoit, E, Boyard, N, Guilminot, E, "Effect of Solvent on PARALOID ${ }^{\circledR}$ B72 and B44 Acrylic Resins Used as Adhesives in Conservation." Herit. Sci., 742 (2019)

18. Chapman, S, Mason, D, "Literature Review: The Use of Paraloid B-72 as a Surface Consolidant for Stained Glass." $J$. Am. Inst. Conserv., 42 381-392 (2003)

19. Ekstedt, J, Studies on the Barrier Properties of Exterior Wood Coatings. Ph.D Thesis. KTH- Royal Institute of Technology, Stockholm, Sweden (2002)

20. Sangaj, N, Malshe, V, "Permeability of Polymers in Protective Organic Coatings." Prog. Org. Coat., 50 28-39 (2004)

21. Carretti, E, Chelazzi, D, Rocchigiani, G, Baglioni, P, Poggi, G, Dei, L, "Interactions Between Nanostructured Calcium Hydroxide and Acrylate Copolymers: Implications in Cultural Heritage Conservation." Langmuir, 29 9881-9890 (2013)

22. Walter, GW, "A Critical Review of the Protection of Metals by Paints." Corr. Sci., 26 (1) 27-38 (1986)

23. Zhang, Y, Li, X, Wei, B, "Environment-Friendly Poly(2ethyl-2-oxazoline) as an Innovative Consolidant for Ancient Wall Paintings." Nanomaterials, 8 (9) 649 (2018)

24. Brasher, DM, Kingsbury, AH, "Electrical Measurements in the Study of Immersed Paint Coatings on Metal. I. Comparison between Capacitance and Gravimetric Methods of Estimating Water-Uptake." J. Appl. Chem., 4 (2) 62-72 (1954)

25. Giorgi, R, Baglioni, M, Berti, D, Baglioni, P, "New Methodologies for the Conservation of Cultural Heritage: 
Micellar Solutions, Microemulsions, and Hydroxide Nanoparticles.”' Acc. Chem. Res., 43 695-704 (2010)

26. Watkinson, D, "Preservation of Metallic Cultural Heritage." In: Cottis, RA (ed.) Shreir's Corrosion, 4th ed., Vol. 4, pp. 3307-3340. Elsevier, London (2010)

27. Product of Viner-Pack Company, http://viner.hu/en/home/

28. EN ISO 7783-1, Determination of Water-Vapor Transmission Rate Part 1: Dish Method for Free Films

29. Huldén, M, Hansen, CM, "Water Permeation in Coatings." Prog. Org. Coat., 13 (3-4) 171-194 (1985)

30. Johansson, P, Lahtinen, K, Kuusipalo, J, Kääriäinen, T, Maydannik, P, Cameron, D, "Atomic Layer Deposition Process for Barrier Applications of Flexible Packaging." In: TAPPI 2010 PLACE Conference. Albuquerque NM, USA (2010)

31. Lahtinen, K, Kotkamo, S, Koskinen, T, Auvinen, S, Kuusipalo, J, "Characterization for Water Vapor Barrier and Heat Sealability Properties of Heat-treated Paperboard/Polylactide Structure." Pack. Tech. Sci., 22 (8) 451-460 (2009)

32. Lahtinen, K, Kuusipalo, J, "Statistical Prediction Model for Water Vapor Barrier of Extrusion-Coated Paper." Tappi J, 7 (9) 8-15 (2008)

33. Kamper, SL, Fennema, O, "Water Vapor Permeability of Edible Bilayer Films.” J. Food Sci., 49 (6) 1478-1481 (1984)

34. Germadios, A, Weller, CL, Gooding, CH, "Measurement Errors in Water Vapor Permeability of Highly Permeable, Hydrophilic Edible Films.' J. Food Eng., 21 (4) 395-409 (1994)

35. McCullough, EA, Kwon, M, Shim, H, “A Comparison of Standard Methods for Measuring Water Vapor Permeability of Fabrics." Meas. Sci. Techol., 14 (8) 1402-1408 (2003)

36. ASTM G31-72, Standard Practice for Laboratory Immersion Corrosion Testing of Metals (2004)

37. Zhang, X, Chen, G, "Immersion and Electrochemical Tests Study on Corrosion Resistance of Chrome-free Chemical Conversion Film on AZ91D $\mathrm{Mg}$ Alloy in $5 \mathrm{wt} \% \mathrm{NaCl}$ Solution." Surf. Rev. Lett., 12 417-424 (2005)

38. Cao-Paz, A, Covelo, A, Farina, J, Nóvoa, XR, Pérez, C, Rodriguez-Pardo, L, "Ingress of Water into Organic Coat- ings: Real-time Monitoring of the Capacitance and Increase in Mass." Prog. Org. Coat., 69 150-157 (2010)

39. Kolek, Z, "Water Absorption by Lacquer Coatings." Pack. Technol. Sci., 9 99-110 (1996)

40. Podany, J, Garland, K, Freeman, W, Rogers, J, "Paraloid B72 as a Structural Adhesive and as a Barrier within Structural Adhesive Bonds: Evaluations of Strength and Reversibility." J. Am. Inst. Conserv., 40 14-33 (2001)

41. Crank, J, The Mathematics of Diffusion. Clarendon Press, New York (1979)

42. Lide, DR (ed.) CRC Handbook of Chemistry and Physics, 85th ed. CRC Press, Boca Raton (2005)

43. Krongauz, VV, Bennett, SE, Ling, MTK, "Kinetics of Water Vapor Diffusion in Resins." J. Therm. Anal. Calorim., 125 231-243 (2016)

44. Wolfe, J, Grayburn, R, Khanjian, H, Heginbotham, A, Phenix, A, "Deconstructing Incralac: A Formulation Study of Acrylic Coatings for the Protection of Outdoor Bronze Sculpture." In: ICOM-CC 18th Triennial Conference. Copenhagen, Denmark (2017)

45. Finšgar, M, Milošev, I, "Inhibition of Copper Corrosion by 1,2,3-benzotriazole: A Review." Corr. Sci., 52 (9) 2737-2749 (2010)

46. Ravichandran, R, Nanjundan, S, Rajendran, N, "Corrosion Inhibition of Brass by Benzotriazole Derivatives in $\mathrm{NaCl}$ Solution." Anti-Corrosion Method M, 52 (4) 226-232 (2005)

47. Hashemi, T, Hogarth, CA, "The Mechanism of Corrosion Inhibition of Copper in $\mathrm{NaCl}$ Solution by Benzotriazole Studied by Electron Spectroscopy." Electrochim. Acta, 33 (8) 1123-1127 (1988)

48. Nilsson, J-O, Törnkvist, C, Liedberg, B, "Photoelectron and Infrared Reflection Absorption Spectroscopy of Benzotriazole Adsorbed on Copper and Cuprous Oxide Surfaces." Appl. Surf. Sci., 37 (3) 306-326 (1989)

Publisher's Note Springer Nature remains neutral with regard to jurisdictional claims in published maps and institutional affiliations. 